\title{
Asymptotic Behavior of Stochastic Strongly Damped Wave Equation with Multiplicative Noise
}

\author{
Zhaojuan Wang1, Shengfan Zhou ${ }^{2}$ \\ ${ }^{1}$ School of Mathematical Science, Huaiyin Normal University, Huaian, China \\ ${ }^{2}$ Department of Mathematics, Zhejiang Normal University, Jinhua, China \\ Email: wangzhaojuan2006@163.com
}

Received 7 August 2015; accepted 11 September 2015; published 15 September 2015

Copyright @ 2015 by authors and Scientific Research Publishing Inc.

This work is licensed under the Creative Commons Attribution International License (CC BY). http://creativecommons.org/licenses/by/4.0/

c) (i) Open Access

\section{Abstract}

In this paper we study the asymptotic dynamics of the stochastic strongly damped wave equation with multiplicative noise under homogeneous Dirichlet boundary condition. We investigate the existence of a compact random attractor for the random dynamical system associated with the equation.

\section{Keywords}

\section{Stochastic Strongly Damped Wave Equation, Random Dynamical System, Random Attractor}

\section{Introduction}

Consider the following stochastic strongly damped wave equation with multiplicative noise:

$$
u_{t t}-\alpha \Delta u_{t}+u_{t}+f(u)-\Delta u=g+c u \circ \frac{\mathrm{d} W}{\mathrm{~d} t} \text { in } U \times[0,+\infty)
$$

with the homogeneous Dirichlet boundary condition

$$
u(x, t)=0 \quad \text { on } \partial U \times[0,+\infty),
$$

and the initial value conditions

$$
u(x, 0)=u_{0}(x) \in H_{0}^{1}(U), \quad u_{t}(x, 0)=u_{1}(x) \in L^{2}(U),
$$

where $\Delta$ is the Laplacian with respect to the variable $x \in U, U \subset \mathbb{R}^{n}$ is a bounded open set with a smooth 
boundary $\partial U ; u=u(x, t)$ is a real function of $x \in U$ and $t \geq 0 ; c \neq 0, \quad \alpha>0$ are strong damping coefficients; o denotes the Stratonovich sense of the stochastic term; $g \in L^{2}(U)$ is a given external force; $f \in C^{1}(\mathbb{R}, \mathbb{R}), f^{\prime}$ are uniformly bounded and there exist $c_{0}, c_{1}, c_{2} \geq 0$ such that

$$
\begin{gathered}
\left|f^{\prime}(u)\right| \leq c_{0}, \quad|f(u)| \leq c_{1}, \quad \forall u \in \mathbb{R}, \\
\left|f^{\prime}(u)-f^{\prime}(v)\right| \leq c_{2}|u-v|, \quad \forall u, v \in \mathbb{R},
\end{gathered}
$$

where $|\cdot|$ denotes the absolute value of number in $\mathbb{R} . W(t)$ is a one-dimensional two-sided real-valued Wiener process on probability space $(\Omega, \mathcal{F}, \mathbb{P})$, where

$$
\Omega=\{\omega \in C(\mathbb{R}, \mathbb{R}): \omega(0)=0\},
$$

the Borel $\sigma$-algebra $\mathcal{F}$ on $\Omega$ is generated by the compact open topology, and $\mathbb{P}$ is the corresponding Wiener measure on $\mathcal{F}$. We identify $\omega(t)$ with $W(t)$, i.e., $W(t)=\omega(t), t \in \mathbb{R}$. When $f(u)=\sin u$ and $\alpha=0$, Equation (1.1) can be regarded as a stochastic perturbed model of a continuous Josephson junction [1], which is stochastic damped sine-Gordon equation [2].

A large amount of studies have been carried out toward the dynamics of a variety of systems related to Equation (1.1). For example, the asymptotical behavior of solutions for deterministic and stochastic wave equations has been studied by many authors, see, e.g. [3]-[27] and the references therein.

In this paper we study the existence of a global random attractor for stochastic strongly damped wave equations with multiplicative noise $c u \circ \frac{\mathrm{d} W}{\mathrm{~d} t}$. The coefficient $c$ of the noise term needs to be suitable small, which is different from that in stochastic strongly damped wave equations with additive noise, this is because the multiplicative noise depends on the state variable $u$ but the additive noise term is independent of $u$.

This paper is organized as follows. In the next section, we recall some basic concepts and properties for general random dynamical systems. In Section 3, we provide some basic settings about Equation (1.1) and show that it generates a random dynamical system in proper function space. Section 4 is devoted to proving the existence of a unique random attractor of the random dynamical system.

\section{Preliminaries}

In this section, we collect some basic knowledge about general random dynamical systems (see [28] [29] for details).

Let $\left(X,\|\cdot\|_{X}\right)$ be a separable Hilbert space with Borel $\sigma$-algebra $\mathcal{B}(X)$. Let $(\Omega, \mathcal{F}, \mathbb{P})$ be a probability space as in Section 1 . Define $\left(\theta_{t}\right)_{t \in \mathbb{R}}$ on $\Omega$ via

$$
\theta_{t} \omega(\cdot)=\omega(\cdot+t)-\omega(t), \quad t \in \mathbb{R}
$$

then $\left(\Omega, \mathcal{F}, \mathbb{P},\left(\theta_{t}\right)_{t \in \mathbb{R}}\right)$ is an ergodic metric dynamical system [28] [29].

In the following, a property holds for $\mathbb{P}$-a.e. $\omega \in \Omega$ means that there is $\Omega_{0} \in \Omega$ with $\mathbb{P}\left(\Omega_{0}\right)=1$ and $\theta_{t} \Omega_{0}=\Omega_{0}$ for $t \in \mathbb{R}$.

Definition 2.1 A continuous random dynamical system on $X$ over $\left(\Omega, \mathcal{F}, \mathbb{P},\left(\theta_{t}\right)_{t \in \mathbb{R}}\right)$ is a mapping

$$
\varphi: \mathbb{R}^{+} \times \Omega \times X \rightarrow X, \quad(t, \omega, u) \mapsto \varphi(t, \omega, u)
$$

which is $\left(\mathcal{B}\left(\mathbb{R}^{+}\right) \times \mathcal{F} \times \mathcal{B}(X), \mathcal{B}(X)\right)$-measurable and satisfies, for $\mathbb{P}$-a.e. $\omega \in \Omega$,

1) $\varphi(0, \omega, \cdot)$ is the identity on $X$;

2) $\varphi(t+s, \omega, \cdot)=\varphi\left(t, \theta_{s} \omega, \varphi(s, \omega, \cdot)\right)$ for all $s, t \geq 0$;

3) $\varphi(t, \omega, \cdot): X \rightarrow X$ is continuous for all $t \geq 0$.

Definition 2.2 (See [29]).

1) A set-valued mapping $\{D(\omega)\}: \Omega \rightarrow 2^{X} \backslash \varnothing, \omega \mapsto D(\omega)$ is said to be a random set if the mapping $\omega \mapsto d(u, D(\omega))$ is measurable for any $u \in X$. If $D(\omega)$ is also closed (compact) for each $\omega \in \Omega,\{D(\omega)\}$ 
is called a random closed (compact) set. A random set $\{D(\omega)\}$ is said to be bounded if there exist $u_{0} \in X$ and a random variable $R(\omega)>0$ such that

$$
D(\omega) \subset\left\{u \in X:\left\|u-u_{0}\right\|_{X} \leq R(\omega)\right\} \text { for all } \omega \in \Omega \text {. }
$$

2) A random set $\{D(\omega)\}$ is called tempered provided for $\mathbb{P}$-a.e. $\omega \in \Omega$,

$$
\lim _{t \rightarrow \infty} \mathrm{e}^{-\beta t} \sup \left\{\|b\|_{X}: b \in D\left(\theta_{-t} \omega\right)\right\}=0 \quad \text { for all } \beta>0 .
$$

3) A random set $\{B(\omega)\}$ is said to be a random absorbing set if for any tempered random set $\{D(\omega)\}$, and $\mathbb{P}$-a.e. $\omega \in \Omega$, there exists $t_{0}(\omega)$ such that

$$
\varphi\left(t, \theta_{-t} \omega, D\left(\theta_{-t} \omega\right)\right) \subset B(\omega) \text { for all } t \geq t_{0}(\omega) .
$$

4) A random set $\left\{B_{1}(\omega)\right\}$ is said to be a random attracting set if for any tempered random set $\{D(\omega)\}$, and $\mathbb{P}$-a.e. $\omega \in \Omega$, we have

$$
\lim _{t \rightarrow \infty} d_{H}\left(\varphi\left(t, \theta_{-t} \omega, D\left(\theta_{-t} \omega\right), B_{1}(\omega)\right)\right)=0,
$$

where $d_{H}$ is the Hausdorff semi-distance given by $d_{H}(G, F)=\sup _{u \in G} \inf _{v \in E}\|u-v\|_{X}$ for any $G, F \subset X$.

5) A random compact set $\{A(\omega)\}$ is said to be a random attractor if it is a random attracting set and $\varphi(t, \omega, A(\omega))=A\left(\theta_{t} \omega\right)$ for $\mathbb{P}$-a.e. $\omega \in \Omega$ and all $t \geq 0$.

Theorem 2.3 (See [29]). Let $\varphi$ be a continuous random dynamical system on $X$ over $\left(\Omega, \mathcal{F}, \mathbb{P},\left(\theta_{t}\right)_{t \in \mathbb{R}}\right)$. If there is a tempered random compact absorbing set $\{B(\omega)\}$ of $\varphi$, then $\{A(\omega)\}$ is a compact random attractor of $\varphi$, where

$$
A(\omega)=\bigcap_{t>0 \tau \geq t} \varphi\left(\tau, \theta_{-\tau} \omega, B\left(\theta_{-\tau} \omega\right)\right), \quad \omega \in \Omega .
$$

Moreover, $\{A(\omega)\}$ is the unique random attractor of $\varphi$.

\section{Stochastic Strongly Damped Wave Equation}

In this section, we outline the basic setting of (1.1)-(1.2) and show that it generates a random dynamical system.

Define an unbounded operator

$$
A: D(A) \equiv H_{0}^{1}(U) \cap H^{2}(U) \rightarrow L^{2}(U), u \mapsto-\Delta u .
$$

Clearly, $A$ is a self-adjoint, positive linear operator with the eigenvalues $\left\{\lambda_{i}\right\}_{i \in \mathbb{N}}$ :

$$
0<\lambda_{1} \leq \lambda_{2} \leq \cdots \leq \lambda_{i} \leq \cdots, \lambda_{i} \rightarrow+\infty(i \rightarrow+\infty) .
$$

It is well known that $-A$ generates an analytic semigroup of bounded linear operators $\left\{\mathrm{e}^{-A t}\right\}_{t \geq 0}$ on $L^{2}(U)$.

Let $E=H_{0}^{1}(U) \times L^{2}(U)$, endowed with the usual norm

$$
\|Y\|_{H_{0}^{1} \times L^{2}}=\left(\|\nabla u\|^{2}+\|v\|^{2}\right)^{\frac{1}{2}} \quad \text { for } Y=(u, v)^{\mathrm{T}} \in E,
$$

where $\|\cdot\|$ denotes the usual norm in $L^{2}(U)$ and $\mathrm{T}$ stands for the transposition.

It is convenient to reduce (1.1) to an evolution equation of the first order in time

$$
\left\{\begin{array}{l}
\frac{\mathrm{d} u}{\mathrm{~d} t}=v, \\
\frac{\mathrm{d} v}{\mathrm{~d} t}=-\alpha A v-A u-v-f(u)+g+c u \circ \frac{\mathrm{d} W}{\mathrm{~d} t}, \\
u(x, 0)=u_{0}(x), \quad v(x, 0)=u_{1}(x), \quad x \in U .
\end{array}\right.
$$


For our purpose, it is convenient to convert the problems (1.1)-(1.2) into a deterministic system with a random parameter, and then show that it generates a random dynamical system.

We now introduce an Ornstein-Uhlenbeck process given by the Brownian motion. Put

$$
z\left(\theta_{t} \omega\right)=-\int_{-\infty}^{0} \mathrm{e}^{s}\left(\theta_{t} \omega\right)(s) \mathrm{d} s, \quad t \in \mathbb{R},
$$

which is called Ornstein-Uhlenbeck process and solves the Itô equation

$$
\mathrm{d} z+z \mathrm{~d} t=\mathrm{d} W(t) .
$$

From [30] [31], it is known that the random variable $z(\omega)$ is tempered, and there is a $\theta_{t}$-invariant set $\Omega_{0} \subset \Omega$ of full $\mathbb{P}$ measure such that $z\left(\theta_{t} \omega\right)$ is continuous in $t$ for every $\omega \in \Omega_{0}$.

Lemma 3.1 (See [7]). For the Ornstein-Uhlenbeck process $z\left(\theta_{t} \omega\right)$ in Equation (3.3), we have the following results

$$
\begin{aligned}
& \lim _{t \rightarrow \infty} \frac{1}{t} \int_{0}^{t}\left|z\left(\theta_{s} \omega\right)\right| \mathrm{d} s=\boldsymbol{E}\left[\left|z\left(\theta_{s} \omega\right)\right|\right]=\frac{1}{\sqrt{\pi}}, \\
& \lim _{t \rightarrow \infty} \frac{1}{t} \int_{0}^{t}\left|z\left(\theta_{s} \omega\right)\right|^{2} \mathrm{~d} s=\boldsymbol{E}\left[\left|z\left(\theta_{s} \omega\right)\right|^{2}\right]=\frac{1}{2}, \\
& \lim _{t \rightarrow \infty} \frac{1}{t} \int_{0}^{t}\left|z\left(\theta_{s} \omega\right)\right|^{4} \mathrm{~d} s=\boldsymbol{E}\left[\left|z\left(\theta_{s} \omega\right)\right|^{4}\right]=\frac{3}{4} .
\end{aligned}
$$

To show that problem (3.2) generates a random dynamical system, we let

$$
\phi_{1}=u, \quad \phi_{2}=v+\varepsilon u-\operatorname{cuz}\left(\theta_{t} \omega\right),
$$

which $\varepsilon$ is a given positive number, then problems (1.1)-(1.2) can be rewritten as the equivalent system with random coefficients but without multiplicative noise on $E$,

$$
\left\{\begin{aligned}
& \frac{\mathrm{d} \phi_{1}}{\mathrm{~d} t}= \phi_{2}-\varepsilon \phi_{1}+c \phi_{1} z\left(\theta_{t} \omega\right), \\
& \frac{\mathrm{d} \phi_{2}}{\mathrm{~d} t}=(\varepsilon-1-\alpha A) \phi_{2}-(\varepsilon(\varepsilon-1-\alpha A)+A) \phi_{1}-f\left(\phi_{1}\right)+g \\
& \quad-\alpha c A \phi_{1} z\left(\theta_{t} \omega\right)-c\left(\phi_{2}-2 \varepsilon \phi_{1}+c \phi_{1} z\left(\theta_{t} \omega\right) z\left(\theta_{t} \omega\right)\right), \\
& \phi_{1}(x, 0)=u_{0}(x), \quad \phi_{2}(x, 0)=u_{1}(x)+\varepsilon u_{0}(x)-c u_{0}(x) z(\omega), \quad x \in U,
\end{aligned}\right.
$$

which has the following vector form

$$
\left\{\begin{array}{l}
\frac{\mathrm{d} \phi}{\mathrm{d} t}=M_{\varepsilon} \phi+F_{\varepsilon}\left(\theta_{t} \omega, \phi\right) \\
\phi_{0}(x, 0)=\left(u_{0}(x), u_{1}(x)+\varepsilon u_{0}(x)-c u_{0}(x) z(\omega)\right)^{\mathrm{T}}, \quad x \in U,
\end{array}\right.
$$

where

$$
\begin{gathered}
\phi=\left(\begin{array}{l}
\phi_{1} \\
\phi_{2}
\end{array}\right), \quad M_{\varepsilon}=\left(\begin{array}{cc}
-\varepsilon I & I \\
\varepsilon(1-\varepsilon) I+(\varepsilon \alpha-1) A & (\varepsilon-1) I-\alpha A
\end{array}\right), \\
F_{\varepsilon}\left(\theta_{t} \omega, \phi\right)=\left(\begin{array}{c}
c \phi_{1} z\left(\theta_{t} \omega\right) \\
-f\left(\phi_{1}\right)+g-\alpha c A \phi_{1} z\left(\theta_{t} \omega\right)-c\left(\phi_{2}-2 \varepsilon \phi_{1}+c \phi_{1} z\left(\theta_{t} \omega\right)\right) z\left(\theta_{t} \omega\right)
\end{array}\right) .
\end{gathered}
$$

We will consider Equation (3.8) or (3.9) for $\omega \in \Omega_{0}$ and write $\Omega_{0}$ as $\Omega$ from now on.

By the classical theory concerning the existence and uniqueness of the solutions [17] [32], one may show that under conditions (1.4)-(1.5), for each $\omega \in \Omega$, problem (3.9) has a unique solution $\phi\left(t, \omega, \phi_{0}\right)$ which is continuous with respect to $\phi_{0}$ in $E$ for all $t \geq 0$. Then the solution mapping

$$
\Phi(t, \omega): \phi_{0} \mapsto \phi\left(t, \omega, \phi_{0}\right) \text {, for } \phi_{0} \in E, t \geq 0 \text { and for all } \omega \in \Omega \text {. }
$$


generates a continuous random dynamical system over $\left(\Omega, \mathcal{F}, \mathbb{P},\left(\theta_{t}\right)_{t \in \mathbb{R}}\right)$ on $E$.

Introduce the homeomorphism $T\left(\varepsilon, \theta_{t} \omega\right)(u, v)^{\mathrm{T}}=\left(u, v+\varepsilon u-\operatorname{cuz}\left(\theta_{t} \omega\right)\right)^{\mathrm{T}}$, whose inverse homeomorphism is $T^{-1}\left(\varepsilon, \theta_{t} \omega\right)(u, v)^{\mathrm{T}}=\left(u, v-\varepsilon u+\operatorname{cuz}\left(\theta_{t} \omega\right)\right)^{\mathrm{T}}, \quad(u, v)^{\mathrm{T}} \in E$. Then the transformation

$$
\Psi(t, \omega)=T\left(\varepsilon, \theta_{t} \omega\right) \Phi(t, \omega) T^{-1}\left(\varepsilon, \theta_{t} \omega\right),
$$

also generates a continuous random dynamical system associated with the problem (3.2) on $E$.

Note that the two random dynamical systems $\Psi$ and $\Phi$ are equivalent. By transformation (3.11), it is easy to see that $\Psi$ has a random attractor provided $\Phi$ possesses a random attractor. Thus, we only need to consider the random dynamical system $\Phi$.

\section{Random Attractor}

In this section, we study the existence of a random attractor. Throughout this section we assume that $\mathscr{P}(E)$ is the collection of all tempered random subsets of $E$ and

$$
|c|<\frac{2 \sigma \sqrt{\pi \lambda_{1} \mu}}{\varepsilon+\sqrt{\lambda_{1} \mu}+\sqrt{\left(\varepsilon+\sqrt{\lambda_{1} \mu}\right)^{2}+2 \sigma \pi\left(\alpha \lambda_{1}+\sqrt{\lambda_{1} \mu}\right)}} .
$$

For our purpose, we introduce a new norm $\|\cdot\|_{E}$ by

$$
\|Y\|_{E}=\left(\mu\left\|A^{\frac{1}{2}} u\right\|^{2}+\|v\|^{2}\right)^{\frac{1}{2}}
$$

for $Y=(u, v)^{\mathrm{T}} \in E$, where $A^{\frac{1}{2}}=\nabla$ and $\mu$ is chosen such that $\mu=1-\alpha \varepsilon \in\left(\frac{1}{2}, 1\right)$ in which $\varepsilon \in(0,1)$ is a small positive number. It is easy to check that $\|\cdot\|_{E}$ is equivalent to the usual norm $\|\cdot\|_{H_{0}^{1} \times L^{2}}$ on $E$ in (3.1). For $Y_{i}=\left(u_{i}, v_{i}\right)^{\mathrm{T}} \in E, \quad i=1,2$, let

$$
\left\langle Y_{1}, Y_{2}\right\rangle_{E}=\mu\left\langle A^{\frac{1}{2}} u_{1}, A^{\frac{1}{2}} u_{2}\right\rangle+\left\langle v_{1}, v_{2}\right\rangle,
$$

where $\langle\cdot, \cdot\rangle$ denotes the inner product on $L^{2}(U)$. By the Poincaré inequality

$$
\left\|A^{\frac{1}{2}} u\right\|^{2} \geq \lambda_{1}\|u\|^{2}, \quad \forall u \in H_{0}^{1}(U),
$$

Equation (4.3) is then positive definite.

Now, we present a property of the operator $M_{\varepsilon}$ in $E$ that plays an important role in this article.

Lemma 4.1 Let $E_{1}=D(A) \times H_{0}^{1}(U)$. There exists a small positive constant $\sigma \in(0, \varepsilon)$ such that for any $Y=(u, v)^{\mathrm{T}} \in E_{1}$,

$$
\left\langle M_{\varepsilon} Y, Y\right\rangle_{E} \leq-\sigma\|Y\|_{E}^{2}-\frac{\alpha}{2}\left\|A^{\frac{1}{2}} v\right\|^{2}-\frac{1}{2}\|v\|^{2} .
$$

The proof of Lemma 4.1 is similar to that of Lemma 1 in [24]. We hence omit it here.

Lemma 4.2 Assume that $g \in H^{1}(U)$, conditions (1.4), (1.5) and (4.1) hold. Then, there exists a random ball $\left\{B_{0}(\omega)\right\} \in \mathscr{P}(E)$ centered at 0 with random radius $\rho(\omega)>0$ such that for any $\{\tilde{B}(\omega)\} \in \mathscr{P}(E)$, there is a $T_{\tilde{B}}(\omega, \rho)>0$ such that for any $\phi_{0}\left(\theta_{-t} \omega\right) \in \tilde{B}\left(\theta_{-t} \omega\right)$ satisfies for $\mathbb{P}$-a.e. $\omega \in \Omega$ and all $t \geq T_{\tilde{B}}(\omega, \rho)$,

$$
\left\|\phi\left(t, \theta_{-t} \omega, \phi_{0}\left(\theta_{-t} \omega\right)\right)\right\|_{E} \leq \rho(\omega) .
$$


Proof. Take the inner product $\langle\cdot, \cdot\rangle_{E}$ of problem (3.9) with $\phi$. By the Cauchy-Schwartz inequality and the Young inequality, we find that

$$
\begin{gathered}
\mu\left\langle c A^{\frac{1}{2}} \phi_{1} z\left(\theta_{t} \omega\right), A^{\frac{1}{2}} \phi_{1}\right\rangle \leq\left|\mu c z\left(\theta_{t} \omega\right)\right| \cdot\left\|A^{\frac{1}{2}} \phi_{1}\right\|^{2}, \\
\left\langle\alpha c A \phi_{1} z\left(\theta_{t} \omega\right), \phi_{2}\right\rangle \leq\left|\alpha c z\left(\theta_{t} \omega\right)\right| \cdot\left\|A^{\frac{1}{2}} \phi_{1}\right\| \cdot\left\|A^{\frac{1}{2}} \phi_{2}\right\| \leq \frac{\alpha}{2}\left|c z\left(\theta_{t} \omega\right)\right|^{2} \cdot\left\|A^{\frac{1}{2}} \phi_{1}\right\|^{2}+\frac{\alpha}{2}\left\|A^{\frac{1}{2}} \phi_{2}\right\|^{2}, \\
\left\langle f\left(\phi_{1}\right), \phi_{2}\right\rangle \leq c_{1}^{2}|U| \cdot\left\|\phi_{2}\right\| \leq c_{1}^{4}|U|^{2}+\frac{1}{4}\left\|\phi_{2}\right\|^{2}, \\
\left\langle g, \phi_{2}\right\rangle \leq\|g\| \cdot\left\|\phi_{2}\right\| \leq\|g\|^{2}+\frac{1}{4}\left\|\phi_{2}\right\|^{2}, \\
\left\langle c \phi_{2} z\left(\theta_{t} \omega\right), \phi_{2}\right\rangle \leq\left|c z\left(\theta_{t} \omega\right)\right| \cdot\left\|\phi_{2}\right\|^{2},
\end{gathered}
$$

where $|U|$ is the volume of the set $U$.

By using the Poincaré inequality (4.4), we have that

$$
\begin{aligned}
& \left\langle 2 c \varepsilon \phi_{1} z\left(\theta_{t} \omega\right), \phi_{2}\right\rangle \leq 2\left|c \varepsilon z\left(\theta_{t} \omega\right)\right| \cdot\left\|\phi_{1}\right\| \cdot\left\|\phi_{2}\right\| \\
& \leq \frac{2\left|c \varepsilon z\left(\theta_{t} \omega\right)\right| \mid}{\sqrt{\lambda_{1}}}\left\|A^{\frac{1}{2}} \phi_{1} \mid \cdot\right\| \phi_{2}\left\|\leq \frac{\left|c \varepsilon z\left(\theta_{t} \omega\right)\right|}{\sqrt{\lambda_{1} \mu}}\right\| \phi \|_{E}^{2}, \\
& \left\langle c^{2} \phi_{1} z^{2}\left(\theta_{t} \omega\right), \phi_{2}\right\rangle \leq\left|c z\left(\theta_{t} \omega\right)\right|^{2} \cdot\left\|\phi_{1}\right\| \cdot\left\|\phi_{2}\right\| \\
& \leq \frac{\left|c z\left(\theta_{t} \omega\right)\right|^{2}}{\sqrt{\lambda_{1}}}\left\|A^{\frac{1}{2}} \phi_{1}\right\| \cdot\left\|\phi_{2}\right\| \leq \frac{\left|c z\left(\theta_{t} \omega\right)\right|^{2}}{2 \sqrt{\lambda_{1} \mu}} \cdot\|\phi\|_{E}^{2} .
\end{aligned}
$$

By all the above inequalities and Lemma 4.1, we have

$$
\frac{\mathrm{d}}{\mathrm{d} t}\|\phi\|_{E}^{2}+2 \sigma\|\phi\|_{E}^{2} \leq\left(2\left(1+\frac{\varepsilon}{\sqrt{\lambda_{1} \mu}}\right)\left|c z\left(\theta_{t} \omega\right)\right|+\left(\frac{\alpha}{\mu}+\frac{1}{\sqrt{\lambda_{1} \mu}}\right)\left|c z\left(\theta_{t} \omega\right)\right|^{2}\right)\|\phi\|_{E}^{2}+2\|g\|^{2}+2 c_{1}^{4}|U|^{2} .
$$

By the Gronwall lemma, we have that, for all $t \geq 0$,

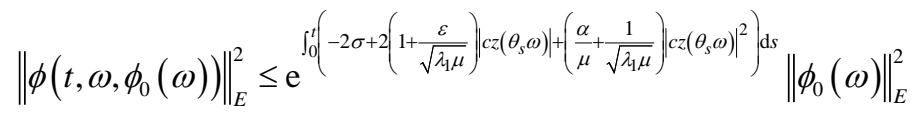

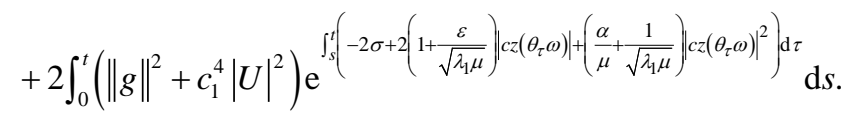

By replacing $\omega$ by $\theta_{-t} \omega$, we get from problem (4.8) that,

$$
\begin{aligned}
& \left\|\phi\left(t, \theta_{-t} \omega, \phi_{0}\left(\theta_{-t} \omega\right)\right)\right\|_{E}^{2} \leq \mathrm{e}^{\left.\int_{0}^{t}\left(-2 \sigma+2\left(1+\frac{\varepsilon}{\sqrt{\lambda_{1} \mu}}\right) \mid c c\left(\theta_{s-t} \omega\right)\right)+\left(\frac{\alpha}{\mu}+\frac{1}{\sqrt{\lambda_{1} \mu}}\right)\left|c c\left(\theta_{s-t} \omega\right)\right|^{2}\right) \mathrm{ds}}\left\|\phi_{0}\left(\theta_{-t} \omega\right)\right\|_{E}^{2}
\end{aligned}
$$

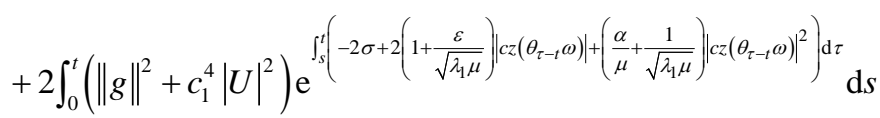

$$
\begin{aligned}
& \leq \mathrm{e}^{\int_{-t}^{0}\left(-2 \sigma+2\left(1+\frac{\varepsilon}{\sqrt{\lambda_{1} \mu}}\right)\left|c c\left(\theta_{s} \omega\right)\right|+\left(\frac{\alpha}{\mu}+\frac{1}{\sqrt{\lambda_{1} \mu}}\right)\left|c z\left(\theta_{s} \omega\right)\right|^{2}\right) \mathrm{ds}}\left\|\phi_{0}\left(\theta_{-t} \omega\right)\right\|_{E}^{2} \\
& +2 \int_{-\infty}^{0}\left(\|g\|^{2}+c_{1}^{4}|U|^{2}\right) \mathrm{e}^{\int_{s}^{0}\left(-2 \sigma+2\left(1+\frac{\varepsilon}{\sqrt{\lambda_{1} \mu}}\right)\left|c z\left(\theta_{\tau} \omega\right)\right|+\left(\frac{\alpha}{\mu}+\frac{1}{\sqrt{\lambda_{1} \mu}}\right)\left|c z\left(\theta_{\tau} \omega\right)\right|^{2}\right) \mathrm{d} \tau} \mathrm{d} s .
\end{aligned}
$$


By inequality (4.1), it is easy to see that

$$
\frac{|c|}{\sqrt{\pi}}\left(1+\frac{\varepsilon}{\sqrt{\lambda_{1} \mu}}\right)+\frac{c^{2}}{4}\left(\frac{\alpha}{\mu}+\frac{1}{\sqrt{\lambda_{1} \mu}}\right)<\sigma .
$$

It then follows from inequality (4.10), Lemma 3.1, $\phi_{0}\left(\theta_{-t} \omega\right) \in \tilde{B}(\omega)$ and $\{\tilde{B}(\omega)\} \in \mathscr{O}(E)$ that

$$
\mathrm{e}^{\int_{-t}^{0}\left(-2 \sigma+\left.2\left(1+\frac{\varepsilon}{\sqrt{\lambda_{1} \mu}}\right)\left|c\left(\theta_{s} \omega\right)+\left(\frac{\alpha}{\mu}+\frac{1}{\sqrt{\lambda_{1} \mu}}\right)\right| c\left(\theta_{s} \omega\right)\right|^{2}\right) \mathrm{ds}}\left\|\phi_{0}\left(\theta_{-t} \omega\right)\right\|_{E}^{2} \rightarrow 0 \text {, as } t \rightarrow \infty .
$$

By Lemma 3.1, inequality (4.10) and $g \in H^{1}(U)$, we have

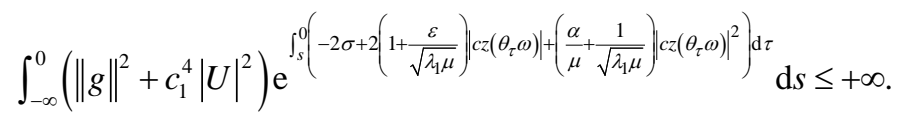

We choose

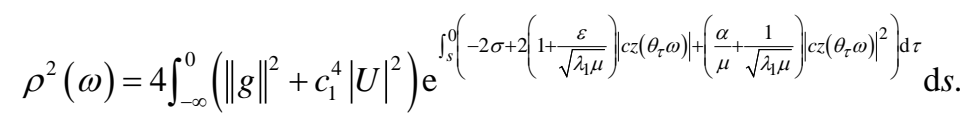

Then, for any tempered random set $\{\tilde{B}(\omega)\} \in \mathscr{P}(E)$, there exists a $T_{\tilde{B}}(\omega, \rho)>0$ such that for any $\phi_{0}\left(\theta_{-t} \omega\right) \in \tilde{B}\left(\theta_{-t} \omega\right)$, satisfies for $\mathbb{P}$-a.e. $\omega \in \Omega, \forall t \geq T_{\tilde{B}}(\omega, \rho)$,

$$
\left\|\phi\left(t, \theta_{-t} \omega, \phi_{0}\left(\theta_{-t} \omega\right)\right)\right\|_{E}^{2} \leq \rho^{2}(\omega) .
$$

So, the proof is completed.

We now construct a random compact attracting set for RDS $\Phi$. For this purpose, we decompose the solution $\phi$ of Equation (3.9) with the initial value $\phi_{0}(\omega)=\left(u_{0}, v_{0}+\varepsilon u_{0}-c u_{0} z(\omega)\right)^{\mathrm{T}}$ into two parts

$\phi=\phi^{a}+\phi^{b}=\left(u^{a}, v^{a}+\varepsilon u^{a}\right)^{\mathrm{T}}+\left(u^{b}, v^{b}+\varepsilon u^{b}-\operatorname{cuz}\left(\theta_{t} \omega\right)\right)^{\mathrm{T}}$, satisfy, respectively

$$
\begin{gathered}
\frac{\mathrm{d} \phi^{a}}{\mathrm{~d} t}=M_{\varepsilon} \phi^{a}, \quad \phi_{0}^{a}=\left(u_{0}, v_{0}+\varepsilon u_{0}\right)^{\mathrm{T}}, \\
\frac{\mathrm{d} \phi^{b}}{\mathrm{~d} t}=M_{\varepsilon} \phi^{b}+F_{\varepsilon}\left(\theta_{t} \omega, \phi\right), \quad \phi_{0}^{b}=\left(0,-c u_{0} z(\omega)\right)^{\mathrm{T}} .
\end{gathered}
$$

Lemma 4.3 Assume that $g \in H^{1}(U)$, conditions (1.4), (1.5) and (4.1) hold. Then, for any $\{\tilde{B}(\omega)\} \in \mathscr{O}(E)$, and $\phi_{0}\left(\theta_{-t} \omega\right) \in \tilde{B}\left(\theta_{-t} \omega\right)$, we have for $\mathbb{P}$-a.e. $\omega \in \Omega$,

$$
\| \phi^{a}\left(t, \theta_{-t} \omega, \phi_{0}^{a}\left(\theta_{-t} \omega\right) \|_{E} \rightarrow 0, \text { as } t \rightarrow \infty,\right.
$$

and there exist a tempered random variable $\rho_{1}(\omega)>0$ and $T_{\tilde{B}}\left(\omega, \rho_{1}\right)>0$ such that for $\mathbb{P}$-a.e. $\omega \in \Omega$ and all $t \geq T_{\tilde{B}}\left(\omega, \rho_{1}\right)$,

$$
\left\|A^{\frac{1}{2}} \phi^{b}\left(t, \theta_{-t} \omega, \phi_{0}^{b}\left(\theta_{-t} \omega\right)\right)\right\|_{E} \leq \rho_{1}(\omega),
$$

where $\phi^{a}$ and $\phi^{b}$ satisfy Equations (4.15), (4.16).

Proof. We first take the inner product $\langle\cdot, \cdot\rangle_{E}$ of Equation (4.15) with $\phi^{a}$. By Lemma 4.1, we obtain

$$
\left\|\phi^{a}\left(t, \theta_{-t} \omega, \phi_{0}^{a}\left(\theta_{-t} \omega\right)\right)\right\|_{E}^{2} \leq \mathrm{e}^{-2 \sigma t}\left\|\phi_{0}^{a}\left(\theta_{-t} \omega\right)\right\|_{E}^{2} .
$$

Then by $\phi_{0}\left(\theta_{-t} \omega\right) \in \tilde{B}\left(\theta_{-t} \omega\right)$ and $\{\tilde{B}(\omega)\} \in \mathscr{D}(E)$, we have

$$
\left\|\phi^{a}\left(t, \theta_{-t} \omega, \phi_{0}^{a}\left(\theta_{-t} \omega\right)\right)\right\|_{E} \rightarrow 0, \text { as } t \rightarrow \infty .
$$


Thus, the first assertion is valid.

Next, we take the inner product $\langle\cdot, \cdot\rangle_{E}$ of Equation (4.16) with $A \phi^{b}$. From the positivity of the operator $A$, we easily obtain

$$
\left\langle M_{\varepsilon} \phi^{b}, A \phi^{b}\right\rangle_{E} \leq-\sigma\left\|A^{\frac{1}{2}} \phi^{b}\right\|_{E}^{2}-\frac{\alpha}{2}\left\|A \phi_{2}^{b}\right\|^{2}-\frac{1}{2}\left\|A^{\frac{1}{2}} \phi_{2}^{b}\right\|^{2} .
$$

By the Cauchy-Schwartz inequality and the Young inequality, we find that

$$
\begin{gathered}
\mu\left\langle c A^{\frac{1}{2}} \phi_{1}^{b} z\left(\theta_{t} \omega\right), A^{\frac{1}{2}} A \phi_{1}^{b}\right\rangle=-\mu\left\langle c A \phi_{1}^{b} z\left(\theta_{t} \omega\right), A \phi_{1}^{b}\right\rangle \leq\left|\mu c z\left(\theta_{t} \omega\right)\right| \cdot\left\|A \phi_{1}^{b}\right\|^{2}, \\
\left\langle\alpha c A \phi_{1}^{b} z\left(\theta_{t} \omega\right), A \phi_{2}^{b}\right\rangle \leq \alpha\left|c z\left(\theta_{t} \omega\right)\right| \cdot\left\|A \phi_{1}^{b}\right\| \cdot\left\|A \phi_{2}^{b}\right\| \leq \frac{\alpha\left|c z\left(\theta_{t} \omega\right)\right|^{2}}{2}\left\|A \phi_{1}^{b}\right\|^{2}+\frac{\alpha}{2}\left\|A \phi_{2}^{b}\right\|^{2}, \\
\left\langle f\left(\phi_{1}^{b}\right), A \phi_{2}^{b}\right\rangle \leq c_{0}^{2}|U| \cdot\left\|A^{\frac{1}{2}} \phi_{2}^{b}\right\| \leq c_{0}^{4}|U|^{2}+\frac{1}{4} \|\left. A^{\frac{1}{2}} \phi_{2}^{b}\right|^{2}, \\
\left\langle g, A \phi_{2}^{b}\right\rangle \leq\left\|A^{\frac{1}{2}} g\right\| \cdot\left\|A^{\frac{1}{2}} \phi_{2}^{b}\right\| \leq\left\|A^{\frac{1}{2}} g\right\|^{2}+\frac{1}{4}\left\|A^{\frac{1}{2}} \phi_{2}^{b}\right\|^{2}, \\
\left\langle c \phi_{2}^{b} z\left(\theta_{t} \omega\right), A \phi_{2}^{b}\right\rangle \leq\left|c z\left(\theta_{t} \omega\right)\right| \cdot\left\|A^{\frac{1}{2}} \phi_{2}^{b}\right\|^{2} .
\end{gathered}
$$

By using inequality (4.4), we have that

$$
\begin{aligned}
\left\langle 2 c \varepsilon \phi_{1}^{b} z\left(\theta_{t} \omega\right), A \phi_{2}^{b}\right\rangle & =\left\langle 2 c \varepsilon A \phi_{1}^{b} z\left(\theta_{t} \omega\right), \phi_{2}^{b}\right\rangle \leq 2 \varepsilon\left|c z\left(\theta_{t} \omega\right)\right| \cdot\left\|A \phi_{1}^{b}\right\| \cdot\left\|\phi_{2}^{b}\right\| \\
& \leq \frac{2 \varepsilon\left|c z\left(\theta_{t} \omega\right)\right|}{\sqrt{\lambda_{1}}} \cdot\left\|A \phi_{1}^{b}\right\| \cdot\left\|A^{\frac{1}{2}} \phi_{2}^{b}\right\| \\
& \leq \frac{\varepsilon\left|c z\left(\theta_{t} \omega\right)\right|}{\sqrt{\lambda_{1} \mu}} \cdot\left\|A^{\frac{1}{2}} \phi^{b}\right\|_{E}^{2}, \\
\left\langle c^{2} \phi_{1}^{b} z^{2}\left(\theta_{t} \omega\right), A \phi_{2}^{b}\right\rangle & =\left\langle c^{2} A \phi_{1}^{b} z^{2}\left(\theta_{t} \omega\right), \phi_{2}^{b}\right\rangle \leq\left|c z\left(\theta_{t} \omega\right)\right|^{2} \cdot\left\|A \phi_{1}^{b}\right\| \cdot\left\|\phi_{2}^{b}\right\| \\
& \leq \frac{\left|c z\left(\theta_{t} \omega\right)\right|^{2}}{\sqrt{\lambda_{1}}} \cdot\left\|A \phi_{1}^{b}\right\| \cdot\left\|A^{\frac{1}{2}} \phi_{2}^{b}\right\| \leq \frac{\left|c z\left(\theta_{t} \omega\right)\right|^{2}}{2 \sqrt{\lambda_{1} \mu}} \cdot\left\|A^{\frac{1}{2}} \phi^{b}\right\|_{E}^{2} .
\end{aligned}
$$

Combining all the above inequalities and inequality (4.21), we have

$$
\begin{aligned}
& \frac{\mathrm{d}}{\mathrm{d} t}\left\|A^{\frac{1}{2}} \phi^{b}\right\|_{E}^{2}+2 \sigma\left\|A^{\frac{1}{2}} \phi^{b}\right\|_{E}^{2} \\
& \leq\left(2\left(1+\frac{\varepsilon}{\sqrt{\lambda_{1} \mu}}\right)\left|c z\left(\theta_{t} \omega\right)\right|+\left(\frac{\alpha}{\mu}+\frac{1}{\sqrt{\lambda_{1} \mu}}\right)\left|c z\left(\theta_{t} \omega\right)\right|^{2}\right)\left\|A^{\frac{1}{2}} \phi^{b}\right\|_{E}^{2}+2\left\|A^{\frac{1}{2}} g\right\|^{2}+2 c_{0}^{4}|U|^{2} .
\end{aligned}
$$

Using the Gronwall lemma, for all $t \geq 0$, we get

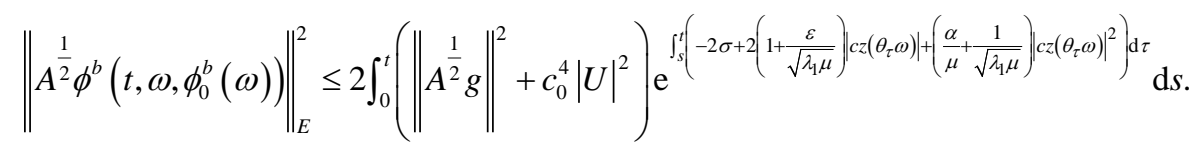

Replacing $\omega$ by $\theta_{-t} \omega$, we get from the above that, 


$$
\begin{aligned}
\left\|A^{\frac{1}{2}} \phi^{b}\left(t, \theta_{-t} \omega, \phi_{0}^{b}\left(\theta_{-t} \omega\right)\right)\right\|_{E}^{2} & \leq 2 \int_{0}^{t}\left(\left\|A^{\frac{1}{2}} g\right\|^{2}+c_{0}^{4}|U|^{2}\right) \mathrm{e}^{\int_{s}^{t}\left(-2 \sigma+2\left(1+\frac{\varepsilon}{\sqrt{\lambda_{1} \mu}}\right)\left|c z\left(\theta_{\tau-t} \omega\right)\right|+\left(\frac{\alpha}{\mu}+\frac{1}{\sqrt{\lambda_{1} \mu}}\right)\left|c z\left(\theta_{\tau-t} \omega\right)\right|^{2}\right) \mathrm{d} \tau} \mathrm{d} s \\
& \leq 2 \int_{-\infty}^{0}\left(\left\|A^{\frac{1}{2}} g\right\|^{2}+c_{0}^{4}|U|^{2}\right) \mathrm{e}^{\int_{s}^{0}\left(-2 \sigma+2\left(1+\frac{\varepsilon}{\sqrt{\lambda_{1} \mu}}\right)\left|c z\left(\theta_{\tau} \omega\right)\right|+\left(\frac{\alpha}{\mu}+\frac{1}{\sqrt{\lambda_{1} \mu}}\right)\left|c z\left(\theta_{\tau} \omega\right)\right|^{2}\right) \mathrm{d} \tau} \mathrm{d} s .
\end{aligned}
$$

By Lemma 3.1, inequality (4.10) and $g \in H^{1}(U)$, we have

$$
\int_{-\infty}^{0}\left(\left\|A^{\frac{1}{2}} g\right\|^{2}+c_{0}^{4}|U|^{2}\right) \mathrm{e}_{s}^{\int_{s}^{0}\left(-2 \sigma+2\left(1+\frac{\varepsilon}{\sqrt{\lambda_{1} \mu}}\right)\left|c z\left(\theta_{\tau} \omega\right)\right|+\left(\frac{\alpha}{\mu}+\frac{1}{\sqrt{\lambda_{1} \mu}}\right)\left|c z\left(\theta_{\tau} \omega\right)\right|^{2}\right) \mathrm{d} \tau} \mathrm{d} s \leq+\infty .
$$

We can choose

$$
\rho_{1}^{2}(\omega)=4 \int_{-\infty}^{0}\left(\left\|A^{\frac{1}{2}} g\right\|^{2}+c_{0}^{4}|U|^{2}\right) \mathrm{e}^{\int_{s}^{0}\left(-2 \sigma+2\left(1+\frac{\varepsilon}{\sqrt{\lambda_{1} \mu}}\right)\left|c z\left(\theta_{\tau} \omega\right)\right|+\left(\frac{\alpha}{\mu}+\frac{1}{\sqrt{\lambda_{1} \mu}}\right)\left|c z\left(\theta_{\tau} \omega\right)\right|^{2}\right) \mathrm{d} \tau} \mathrm{d} s,
$$

then the second assertion is valid.

By Lemma 4.2 and Lemma 4.3, for any $\{\tilde{B}(\omega)\} \in \mathscr{S}(E), \phi_{0}\left(\theta_{-t} \omega\right) \in \tilde{B}\left(\theta_{-t} \omega\right), \quad t \geq T_{\tilde{B}}(\omega, \rho)+T_{\tilde{B}}\left(\omega, \rho_{1}\right)$, and for some constant $\kappa>0$, we have for $\mathbb{P}$-a.e. $\omega \in \Omega$,

$$
\left\|\phi^{b}\left(t, \theta_{-t} \omega, \phi_{0}^{b}\left(\theta_{-t} \omega\right)\right)\right\|_{E_{1}} \leq \kappa\left(\rho(\omega)+\rho_{1}(\omega)\right),
$$

where $E_{1}=D(A) \times H_{0}^{1}(U)$. Let $\{B(\omega)\} \in \mathscr{P}(E)$ be a closed ball of $E$ :

$$
B(\omega)=\left\{\rho(\omega) \in E:\|\rho(\omega)\|_{E_{1}} \leq \kappa\left(\rho(\omega)+\rho_{1}(\omega)\right)\right\} .
$$

Then, by the compact embedding of $E_{1}$ into $E,\{B(\omega)\}$ is compact in $E$.

Note that

$$
\phi\left(t, \theta_{-t} \omega, \phi_{0}\left(\theta_{-t} \omega\right)\right)=\phi^{a}\left(t, \theta_{-t} \omega, \phi_{0}^{a}\left(\theta_{-t} \omega\right)\right)+\phi^{b}\left(t, \theta_{-t} \omega, \phi_{0}^{b}\left(\theta_{-t} \omega\right)\right) .
$$

Then by Lemma 4.3 and inequality (4.27), we have for $\mathbb{P}$-a.e. $\omega \in \Omega$,

$$
d_{H}\left(\phi\left(t, \theta_{-t} \omega, \tilde{B}\left(\theta_{-t} \omega\right)\right), B(\omega)\right) \rightarrow 0 \text { as } t \rightarrow+\infty .
$$

which implies that $\{B(\omega)\}$ is a random compact attracting set for $\Phi$. It follows from Equations (4.13) and (4.26) that $\{B(\omega)\}$ is tempered. Thus by Theorem 2.3, the main result of this section can now be stated as follows.

Theorem 4.4 Assume that $g \in H^{1}(U)$, conditions (1.4), (1.5) and (4.1) hold. Then, the random dynamical system $\Phi$ has a unique compact random attractor. $/(\omega)$ in $E$, where

$$
\mathscr{C}(\omega)=\bigcap_{t>0} \overline{\phi \geq t} \phi\left(\tau, \theta_{-\tau} \omega, B\left(\theta_{-\tau} \omega\right)\right), \quad \omega \in \Omega,
$$

in which $\{B(\omega)\}$ is a tempered random compact attracting set for $\Phi$.

\section{Supported}

The authors are supported by National Natural Science Foundation of China (Nos. 11326114, 11401244, 11071165 and 11471290); Natural Science Research Project of Ordinary Universities in Jiangsu Province (No. 14KJB110003); the Foundation of Zhejiang Normal University (No. ZC304011068).

\section{References}

[1] Lomdahl, P.S., Soerensen, O.H. and Christiansen, P. L. (1982) Soliton Excitations in Josephson Tunnel Junctions. 
Physical Review B, 25, 5337-5348.

[2] Shen, Z.W., Zhou, S.F. and Shen, W.X. (2010) One-Dimensional Random Attractor and Rotation Number of the Stochastic Damped Sine-Gordon Equation. Journal of Differential Equations, 248, 1432-1457. http://dx.doi.org/10.1016/j.jde.2009.10.007

[3] Chen, F.X., Guo, B.L. and Wang, P. (1998) Long Time Behavior of Strongly Damped Nonlinear Wave Equations. Journal of Differential Equations, 147, 231-241. http://dx.doi.org/10.1006/jdeq.1998.3447

[4] Chow, P.-L. (2002) Stochastic Wave Equation with Polynomial Nonlinearity. The Annals of Applied Probability, 12, 361-381. http://dx.doi.org/10.1214/aoap/1015961168

[5] Ghidaglia, J.M. and Marzocchi, A. (1991) Longtime Behavior of Strongly Damped Nonlinear Wave Equations, Global Attractors and Their Dimension. SIAM Journal on Mathematical Analysis, 22, 879-895. http://dx.doi.org/10.1137/0522057

[6] Kalantarov, V. and Zelik, S. (2009) Finite-Dimensional Attractors for the Quasi-Linear Strongly-Damped Wave Equation. Journal of Differential Equations, 247, 1120-1155. http://dx.doi.org/10.1016/j.jde.2009.04.010

[7] Fan, X.M. (2006) Attractors for a Damped Stochastic Wave Equation of Sine-Gordon Type with Sublinear Multiplicative Noise. Stochastic Analysis and Applications, 24, 767-793. http://dx.doi.org/10.1080/07362990600751860

[8] Fan, X.M. (2004) Random Attractor for a Damped Sine-Gordon Equation with White Noise. Pacific Journal of Mathematics, 216, 63-76. http://dx.doi.org/10.2140/pjm.2004.216.63

[9] Fan, X.M. and Wang, Y. (2007) Fractal Dimensional of Attractors for a Stochastic Wave Equation with Nonlinear Damping and White Noise. Stochastic Analysis and Applications, 25, 381-396. http://dx.doi.org/10.1080/07362990601139602

[10] Fan, X.M. (2008) Random Attractors for Damped Stochastic Wave Equations with Multiplicative Noise. International Journal of Mathematics, 19, 421-437. http://dx.doi.org/10.1142/S0129167X08004741

[11] Li, H.Y. and Zhou, S.F. (2007) One-Dimensional Global Attractor for Strongly Damped Wave Equations. Communications in Nonlinear Science and Numerical Simulation, 12, 784-793. http://dx.doi.org/10.1016/j.cnsns.2005.06.007

[12] Li, H.Y. and Zhou, S.F. (2008) On Non-Autonomous Strongly Damped Wave Equations with a Uniform Attractor and Some Averaging. Journal of Mathematical Analysis and Applications, 341, 791-802. http://dx.doi.org/10.1016/j.jmaa.2007.10.051

[13] Lu, K.N. and Schmalfuß, B. (2007) Invariant Manifolds for Stochastic Wave Equations. Journal of Differential Equations, 236, 460-492. http://dx.doi.org/10.1016/j.jde.2006.09.024

[14] Lv, Y. and Wang, W. (2008) Limiting Dynamics for Stochastic Wave Equations. Journal of Differential Equations, 244, 1-23. http://dx.doi.org/10.1016/j.jde.2007.10.009

[15] Massatt, P. (1983) Limiting Behavior for Strongly Damped Nonlinear Wave Equations. Journal of Differential Equations, 48, 334-349. http://dx.doi.org/10.1016/0022-0396(83)90098-0

[16] Pata, V. and Zelik, S. (2006) Smooth Attractors for Strongly Damped Wave Equations. Nonlinearity, 19, $1495-1506$. http://dx.doi.org/10.1088/0951-7715/19/7/001

[17] Temam, R. (1988) Infinite Dimensional Dynamical Systems in Mechanics and Physics. Springer, Berlin. http://dx.doi.org/10.1007/978-1-4684-0313-8

[18] Wang, B.X. and Gao, X.L. (2009) Random Attractors for Wave Equations on Unbounded Domains. Discrete and Continuous Dynamical Systems, Special, 800-809.

[19] Yang, M.H. and Sun, C.Y. (2009) Attractors for Strongly Damped Wave Equations. Nonlinear Analysis: Real World Applications, 10, 1097-1100. http://dx.doi.org/10.1016/j.nonrwa.2007.12.001

[20] Yang, M.H. and Sun, C.Y. (2010) Exponential Attractors for the Strongly Damped Wave Equations. Nonlinear Analysis: Real World Applications, 11, 913-919. http://dx.doi.org/10.1016/j.nonrwa.2009.01.022

[21] Yang, M.H. and Sun, C.Y. (2009) Dynamics of Strongly Damped Wave Equations in Locally Uniform Spaces: Attractors and Asymptotic Regularity. Transactions of the American Mathematical Society, 361, 1069-1101. http://dx.doi.org/10.1090/S0002-9947-08-04680-1

[22] Yang, M.H., Duan, J.Q. and Kloeden, P. (2011) Asymptotic Behavior of Solutions for Random Wave Equations with Nonlinear Damping and White Noise. Nonlinear Analysis: Real World Applications, 12, 464-478. http://dx.doi.org/10.1016/j.nonrwa.2010.06.032

[23] Zhou, S.F. (1999) Dimension of the Global Attractor for Strongly Damped Nonlinear Wave Equation. Journal of Mathematical Analysis and Applications, 233, 102-115. http://dx.doi.org/10.1006/jmaa.1999.6269

[24] Zhou, S.F. (1999) Global Attractor for Strongly Damped Nonlinear Wave Equation. Functional Differential Equations, 6, 451-470. 
[25] Zhou, S.F. and Fan, X.M. (2002) Kernel Sections for Non-Autonomous Strongly Damped Wave Equations. Journal of Mathematical Analysis and Applications, 275, 850-869. http://dx.doi.org/10.1016/S0022-247X(02)00437-7

[26] Zhou, S.F. (2003) Attractors for Strongly Damped Wave Equations with Critical Exponent. Applied Mathematics Letters, 16, 1307-1314. http://dx.doi.org/10.1016/S0893-9659(03)90134-0

[27] Zhou, S.F., Yin, F.Q. and Ouyang, Z.G. (2005) Random Attractor for Damped Nonlinear Wave Equations with White Noise. SIAM Journal on Applied Dynamical Systems, 4, 883-903. http://dx.doi.org/10.1137/050623097

[28] Arnold, L. (1998) Random Dynamical Systems. Springer-Verlag, New York and Berlin. http://dx.doi.org/10.1007/978-3-662-12878-7

[29] Chueshov, I. (2002) Monotone Random Systems Theory and Applications. Springer-Verlag, New York. http://dx.doi.org/10.1007/b83277

[30] Bates, P.W., Lu, K.N. and Wang, B.X. (2009) Random Attractors for Stochastic Reaction-Diffusion Equations on Unbounded Domains. Journal of Differential Equations, 246, 845-869. http://dx.doi.org/10.1016/j.jde.2008.05.017

[31] Duan, J.Q., Lu, K.N. and Schmalfuss, B. (2003) Invariant Manifolds for Stochastic Partial Differential Equations. The Annals of Probability, 31, 2109-2135. http://dx.doi.org/10.1214/aop/1068646380

[32] Pazy, A. (1983) Semigroup of Linear Operators and Applications to Partial Differential Equations. Springer-Verlag, New York. http://dx.doi.org/10.1007/978-1-4612-5561-1 